\title{
Long-Term Efficacy and Safety of Add-On Therapy of Sitagliptin to a Very Small Dose of Glimepiride Versus a Small Dose of Glimepiride Over Eighteen Months
}

\author{
Masako Hatano ${ }^{\mathrm{a}}$, Ikuo Inoue ${ }^{\mathrm{a}}$, Susumu Kurihara ${ }^{\mathrm{b}}$, Hiraku Ono ${ }^{\mathrm{c}}$, Masafumi Matsuda ${ }^{\mathrm{d}}$, \\ Masafumi Kakei ${ }^{\mathrm{e}}$, Toshihiko Inukai ${ }^{\mathrm{f}}$, Yoshimasa Aso ${ }^{\mathrm{g}}$, Shigehiro Katayama ${ }^{\mathrm{h}}$ i
}

\begin{abstract}
Background: In patients associated with type 2 diabetes mellitus, sulfonylureas (SUs) are being prescribed less frequently, and in smaller doses, to avoid hypoglycemia and body weight gain. On the other hand, since dipeptidyl peptidase-4 (DPP-4) inhibitors only infrequently induce hypoglycemia and weight gain, more than $60 \%$ or $70 \%$ of Japanese patients associated with type 2 diabetes mellitus are currently being treated with DPP-4 inhibitors. However, the longterm effects of DPP-4 inhibitors on glycemic control and $\beta$-cell function have not been thoroughly elucidated.
\end{abstract}

Methods: The present study was designed to compare the long-term efficacy and safety of a daily administration of $50 \mathrm{mg}$ sitagliptin added to a very small dose of glimepiride, SUs, of $1.1 \mathrm{mg}$ (SIT group), versus a small dose of glimepiride added by $1 \mathrm{mg}$ to its basal dosage, i.e., $2.1 \mathrm{mg}$ (SU group) up to 18 months in Japanese patients with type 2 diabetes mellitus. Sixty patients, aged 20 to 75 years with hemoglobin A1c (HbA1c) between $7.4 \%$ and $9.0 \%$ on glimepiride with or without metformin, were randomized into two groups. If the target

Manuscript submitted October 22, 2019, accepted November 12, 2019

aDepartment of Endocrinology and Diabetes, Faculty of Medicine, Saitama Medical University, Iruma-gun, Saitama 350-0495, Japan

bDepartment of Endocrinology and Diabetes, Saitama International Medical Center, Saitama Medical University, Hidaka, Saitama 350-1298, Japan

'Department of Diabetes, Metabolism and Endocrinology, Faculty of Medicine, Chiba University, Chiba 260-8677, Japan

${ }^{d}$ Department of Endocrinology and Diabetes, Saitama Medical Center, Saitama Medical University, Kawagoe, Saitama 350-8550, Japan

eDepartment of Diabetes, Saitama Citizens' Medical Center, Oomiya, Saitama 331-0054, Japan

fDepartment of Endocrinology and Metabolism-Hematology, Internal Medicine, Saitama Medical Center, Dokkyo Medical University, Koshigaya, Saitama 343-8555, Japan

gDepartment of Endocrinology and Metabolism, School of Medicine, Dokkyo Medical University, Shimotsuga-gun, Tochigi 321-0293, Japan

hKawagoe Clinic, Saitama Medical University, Kawagoe, Saitama 350-1123, Japan

${ }^{\mathrm{i} C}$ Corresponding Author: Shigehiro Katayama, Kawagoe Clinic, Saitama Medical University, 21-7 Wakitahoncho, Kawagoe, Saitama 350-1123, Japan. Email: skataya@saitama-med.ac.jp

doi: https://doi.org/10.14740/jem621
(HbA1c $\leq 6.9 \%$ or fasting plasma glucose level $\leq 130 \mathrm{mg} / \mathrm{dL}$ ) was not achieved, sitagliptin or glimepiride, respectively, was increased.

Results: After 18 months, HbAlc had significantly decreased to 7.1$7.2 \%$ in both groups $(\mathrm{P}<0.01)$. Plasma levels of insulin and proinsulin remained unchanged during the study. However, the proinsulin/insulin ratio was significantly lower after 6 months and thereafter only in the SU group. Homeostasis model assessment- $\beta$ cell (HOMA- $\beta$ ) demonstrated a significant augmentation at some points during the study in both groups. No severe hypoglycemic episodes or body weight gain were seen in either group.

Conclusions: Add-on therapy of $50 \mathrm{mg}$ of sitagliptin to a very small dose of glimepiride at 1.1 to $1.2 \mathrm{mg} /$ day, or a small dose of glimepiride at $2.1 \mathrm{mg} /$ day, proved effective for improving or maintaining glycemic control without deterioration of $\beta$-cell function over 18 months.

Keywords: Type 2 diabetes mellitus; DPP-4 inhibitor; Sulfonylurea; $\beta$-cell function

\section{Introduction}

Type 2 diabetes mellitus (T2DM), due to its progressive nature, requires a succession of different treatment options. Oral antidiabetic therapy loses efficacy over time [1], so polypharmacy with multiple mechanisms of action is usually necessary to achieve and maintain long-term glycemic control. Sulfonylureas (SUs) are widely used in Japan as first- or second-line treatments; however, SU therapy is associated with weight gain and a substantial risk of hypoglycemia. This threat is exacerbated when clinical doses are escalated to counteract the loss of glycemic control resulting from secondary failure [2]. Sitagliptin, a selective dipeptidyl peptidase-4 (DPP-4) inhibitor, is a medication for patients with T2DM that improves glycemic control through enhancement of the incretin axis [3, $4]$, i.e., inhibition of degradation of incretins, glucagon-like peptide 1 (GLP-1) and gastric inhibitory polypeptide (GIP) through DPP-4. The prolonged activity of intact GLP-1 by DPP-4 inhibition is likely to both improve glucose-induced insulin secretion and to decrease glucagon levels, resulting in the maintenance of $\beta$-cell function [5-10]. In 2009, sitagliptin was 


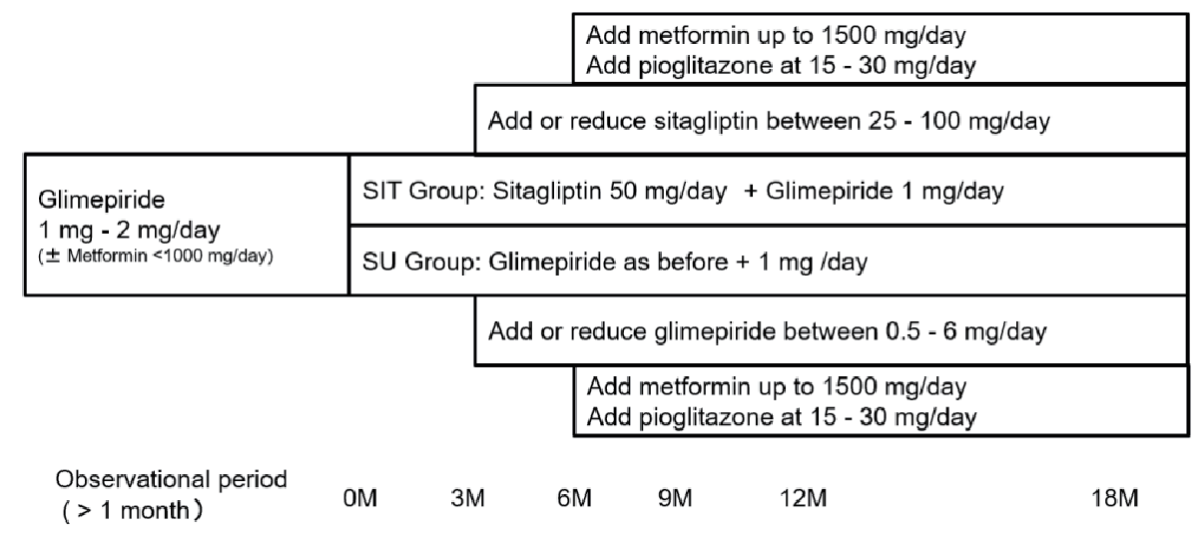

Figure 1. Study design.

the first DPP-4 inhibitor to be approved in Japan as a monotherapy as well as an additional therapy to other hypoglycemic agents in adults with T2DM and inadequate glycemic control.

SUs are being prescribed less frequently, and in smaller doses, to avoid hypoglycemia and body weight gain. For example, although glimepiride was administered up to $6 \mathrm{mg} /$ day in the past 10 - 15 years, the current daily usual doses are from 0.5 to 2 or $3 \mathrm{mg}$. On the other hand, since DPP-4 inhibitors only infrequently induce hypoglycemia and weight gain, more than $60 \%$ or $70 \%$ of Japanese T2DM patients are currently being treated with DPP-4 inhibitors. However, the long-term effects of DPP-4 inhibitors on glycemic control and $\beta$-cell function have not been thoroughly elucidated. The present study was designed, therefore, to compare the long-term efficacy and safety of sitagliptin added to a very small dosage of glimepiride, versus a small increment of $1 \mathrm{mg}$ of glimepiride to its basal dosage, for effect on glycemic control, secretion of proinsulin and insulin, and homeostasis model assessment- $\beta$ cell (HOMA- $\beta$ ) reflecting $\beta$-cell function, up to 18 months in Japanese patients with T2DM.

\section{Patients and Methods}

\section{Patients}

Male and female Japanese patients with T2DM aged 20 - 75 years were eligible to participate if they had inadequate glycemic control of hemoglobin A1c $(\mathrm{HbA} 1 \mathrm{c})$, ranging between $7.4 \%$ and $9.0 \%$ on screening, and had been taking either oral hypoglycemic agents or glimepiride at $1-2 \mathrm{mg} /$ day with or without metformin at up to $1,000 \mathrm{mg} /$ day for the past 1 month or longer. Subjects undergoing treatment with insulin, type 1 diabetics, subjects with a history of diabetic ketoacidosis and/ or hyperglycemic coma, subjects with severe infectious disease or wounds, subjects for whom a surgical operation was scheduled, subjects who were pregnant or were attempting to become pregnant, and subjects with elevated serum creatinine of more than $1.5 \mathrm{mg} / \mathrm{dL}$ were excluded from the study. Also excluded were individuals who had suffered serious adverse events while taking DPP-4 inhibitors and/or glimepiride, and those with other conditions that led the doctors in charge to decide that they were ineligible for this study.

\section{Study design}

The present study was a two-arm, randomized, open-label study (3S: Saitama Sitagliptin Study). After giving their written informed consent, patients were randomized into two groups via an interactive web response system. As shown in Figure 1, in one group (SIT), $50 \mathrm{mg}$ of sitagliptin was administered daily after breakfast with a reduction in glimepiride dosage by $1 \mathrm{mg} /$ day. In the other group (SU), $1 \mathrm{mg}$ of glimepiride was added to the previous daily dosage after breakfast. In both groups, metformin was administered at the same dose during the screening period. The target of glycemic control was HbA1c of less than $6.9 \%$ or a fasting plasma glucose (FPG) level of less than $130 \mathrm{mg} / \mathrm{dL} 3$ months after initiation of the study. If the target glycemic control was not achieved, sitagliptin was increased to $100 \mathrm{mg} /$ day in the SIT group, and glimepiride, up to $6.0 \mathrm{mg} /$ day in the SU group. If the glycemic target was not obtained after another 3 months, metformin was added or increased up to $1,500 \mathrm{mg} /$ day and/or pioglitazone was added at a dose of 15 to $30 \mathrm{mg} /$ day in both groups. If hypoglycemia occurred, sitagliptin was reduced up to $25 \mathrm{mg}$ /day in the SIT group, and glimepiride, up to $0.5 \mathrm{mg} /$ day in the SU group.

Blood samples were collected after an overnight fast to undergo assessment of $\mathrm{HbA1c}, \mathrm{FPG}$, insulin, proinsulin and Cpeptide (CPR) levels at the beginning of the study, i.e., time 0 just before the first dose of study medication, and at 3 months, 6 months, 12 months and 18 months after initiation of the study. Urine samples were also collected to determine urinary albumin and creatinine excretion with the same time schedule. These parameters, except FPG, were determined at a central laboratory (SRL Ltd, Tokyo). Routine laboratory tests, such as hematology, blood chemistry including FPG, and urinalysis, were performed at each hospital with each visit.

Primary endpoints were CPR levels, proinsulin/insulin $(\mathrm{PI} / \mathrm{I})$ ratios and HOMA- $\beta$ to estimate the pancreatic $\beta$-cell function. Secondary endpoints were glycemic control such as $\mathrm{HbA} 1 \mathrm{c}$ and FPG. Changes in estimated glomerular filtration rate (eGFR) and urinary albumin/creatinine ratio (UACR) 
were also analyzed as secondary endpoints. The eGFR was calculated using serum creatinine levels and age according to the Modification of Diet in Renal Disease (MDRD) formula modified for the Japanese population [11]. Safety and tolerability were evaluated at each visit from adverse event (AE) reports, vital signs, and laboratory tests including hematology, blood chemistry and urinalysis.

The study was conducted in compliance with the protocol, the Helsinki Declaration, and the Ethical Guidelines for Clinical Studies released by the Ministry of Health, Labour, and Welfare. The study was approved by Saitama Medical University Hospital's Institutional Review Board on August 17, 2010 (Results Notification Number: 10-019) and registered with UMIN as R000004713. The study was started on December 2010 and scheduled to end on June 2013 in seven sites. However, because of difficulties in recruitment of the patients treated only with SU, the registration period was elongated up to June 2014, and therefore the study was ended on December 2016.

\section{Statistical analyses}

A sample size of 210 (105/treatment arm) was calculated for detecting a significant difference in one of the primary endpoints, i.e. PI/I ratio, with a two-sided significance level of 0.05 and $80 \%$ power assuming a drop-out rate to be $10 \%$. All data are expressed as mean \pm standard deviation (SD) except where specified. The primary and secondary efficacy analyses were performed in the per protocol set. Repeated measures analysis of variance (ANOVA) was used to compare changes in $\mathrm{C}$-peptide, PI/I ratio, HOMA- $\beta, \mathrm{FPG}, \mathrm{HbA} 1 \mathrm{c}$ and weight over time between the groups. If between-group and/or intragroup differences showed statistical significance $(\mathrm{P}<0.05)$, the changes were compared to the basal values in each group using the paired Student's $t$-test. Missing values were imputed using the last observation carried forward (LOCF) method. Safety analyses used the full analysis set that included all patients with a baseline and at least one post-baseline assessment. For overall AEs, drug-related AEs, hypoglycemia, and selected gastrointestinal-related AEs (i.e., nausea, vomiting and diarrhea), comparisons between groups were conducted using Fisher's exact test. All comparisons were conducted using SAS version 9.2 (SAS Institute Inc., Cary, NC, USA). Data with $\mathrm{P}$ values of $<0.05$ were regarded as significant.

\section{Results}

Of the 62 patients screened for participation in this study, two were not randomized, because one withdrew his/her informed consent, and the other had glycemic control of HbA1c of less than $7.4 \%$ at the beginning of the study. The 60 remaining patients were therefore randomized into two groups, with 30 patients in each. The basal patients' characteristics are shown in Table 1. The mean age, percentage of males, body weight, body mass index (BMI) and duration of diabetes mellitus did not differ between the two groups. The initial values in $\mathrm{HbAlc}$, FPG, systolic blood pressure (SBP), diastolic blood pressure
Table 1. The Patients' Basal Characteristics

\begin{tabular}{|c|c|c|}
\hline & SIT group & SU group \\
\hline Age (years) & $65.8 \pm 8.6$ & $64.7 \pm 6.9$ \\
\hline $\operatorname{Sex}(\%)$ & $16(53.3 \%)$ & $17(56.7 \%)$ \\
\hline Body weight (kg) & $63.1 \pm 12.0$ & $64.5 \pm 9.4$ \\
\hline BMI & $24.0 \pm 3.4$ & $25.4 \pm 3.3$ \\
\hline \multicolumn{3}{|l|}{ Duration of diabetes mellitus } \\
\hline$<1$ year & $1(3.3 \%)$ & $1(3.3 \%)$ \\
\hline 1 to $<5$ years & $5(16.7 \%)$ & $5(16.7 \%)$ \\
\hline 5 to $<10$ years & $10(33.3 \%)$ & $5(16.7 \%)$ \\
\hline$>10$ years & $12(40.0 \%)$ & $15(50.0 \%)$ \\
\hline Not known & $2(6.7 \%)$ & $4(13.3 \%)$ \\
\hline HbA1c (\%) & $7.8 \pm 1.1$ & $7.7 \pm 0.6$ \\
\hline $\mathrm{FPG}(\mathrm{mg} / \mathrm{dL})$ & $162.4 \pm 39.9$ & $153.2 \pm 27.0$ \\
\hline $\mathrm{SBP}(\mathrm{mm} \mathrm{Hg})$ & $127.2 \pm 10.2$ & $131.9 \pm 16.8$ \\
\hline DBP (mm Hg) & $72.1 \pm 9.6$ & $76.0 \pm 10.3$ \\
\hline LDL-C (mg/dL) & $111.4 \pm 31.8$ & $113.8 \pm 23.5$ \\
\hline TG (mg/dL) & $142.4 \pm 73.6$ & $132.1 \pm 77.5$ \\
\hline HDL-C (mg/dL) & $52.5 \pm 13.3$ & $52.5 \pm 10.7$ \\
\hline Smoking (\%) & $5(16.7 \%)$ & $1(3.3 \%)$ \\
\hline Drinking habit (\%) & $7(23.3 \%)$ & $7(23.3 \%)$ \\
\hline \multicolumn{3}{|l|}{ Comorbidities } \\
\hline Hypertension & $17(56.7 \%)$ & $22(73.3 \%)$ \\
\hline Dyslipidemia & $13(43.3 \%)$ & $13(43.3 \%)$ \\
\hline Hyperuricemia & $2(6.7 \%)$ & $1(3.3 \%)$ \\
\hline Myocardial infarction & $0(0 \%)$ & $1(3.3 \%)$ \\
\hline Angina pectoris & $1(3.3 \%)$ & $0(0)$ \\
\hline Arteriosclerosis obliterans & $0(0 \%)$ & $1(3.3 \%)$ \\
\hline
\end{tabular}

BMI: body mass index; HbA1c: hemoglobin A1c; FPG: fasting plasma glucose; SBP: systolic blood pressure; DBP: diastolic blood pressure; LDL-C: low-density lipoprotein cholesterol; TG: triglycerides; HDL-C: high-density lipoprotein cholesterol.

(DBP), low-density lipoprotein cholesterol (LDL-C), triglycerides (TG) and high-density lipoprotein cholesterol (HDL-C) did not differ between the two groups; the percentage of patients who had the habit of smoking or drinking did not differ between the groups; the percentage of patients who had comorbidities such as hypertension, dyslipidemia, hyperuricemia, myocardial infarction, angina pectoris and arteriosclerosis obliterans was also the same in both groups. The daily dosage of glimepiride in the SIT group and the SU group was $1.2 \pm$ $0.5 \mathrm{mg}$ and $2.1 \pm 0.8 \mathrm{mg}$, respectively. Four subjects in the SIT group discontinued the study: one to begin treatment by insulin injection, and three for AEs (hypoglycemia in one, headache in one and skin rush in one); three subjects in the SU group discontinued the study, with one for initiation of insulin injection, one for poor glycemic control with the hypoglycemic drugs used in the study protocol, and one for AE (hypoglycemia in one). As a result, 26 subjects in the SIT group and 27 in the SU 

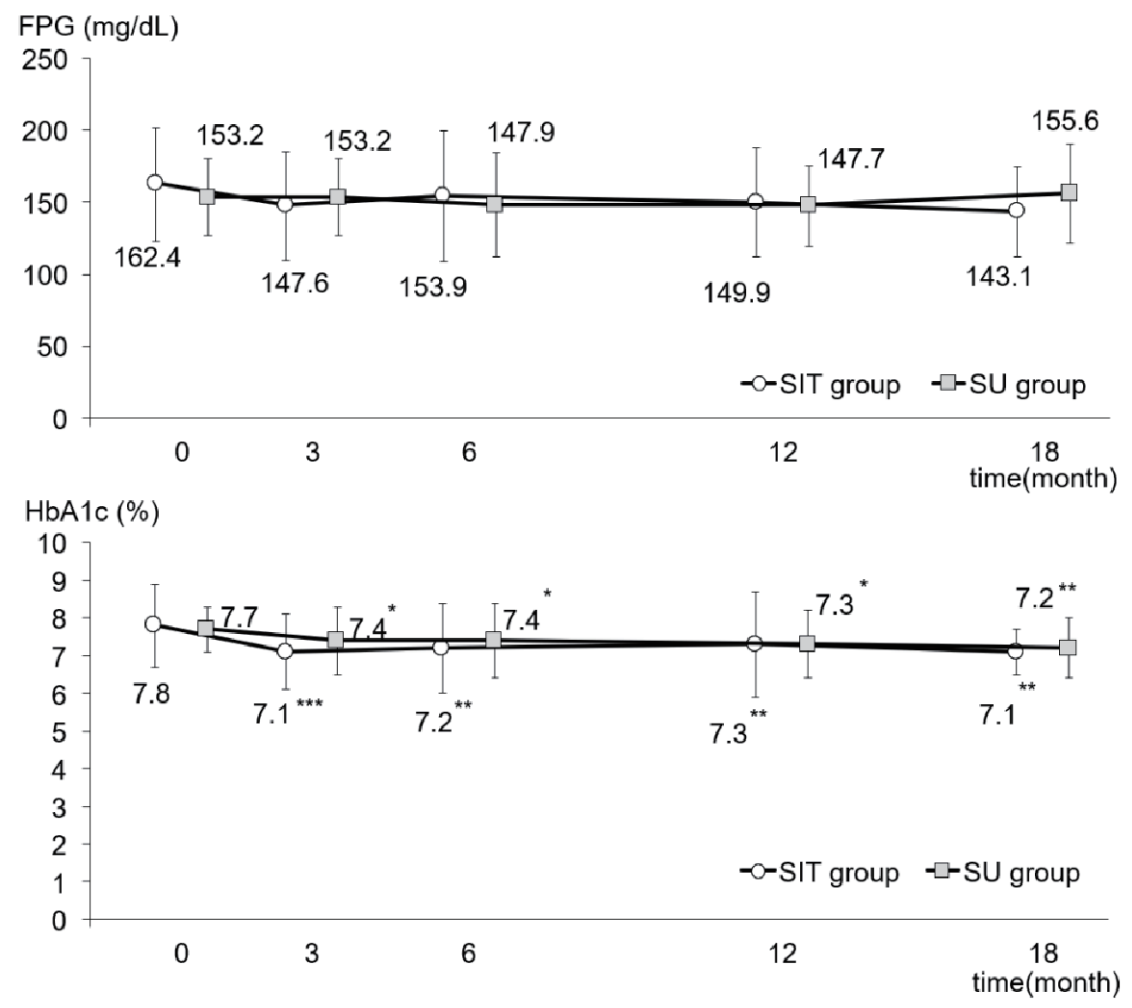

Figure 2. FPG and $\mathrm{HbA} 1 \mathrm{c}$ levels during the study in the SIT and SU groups. All data were presented as mean \pm standard deviation (SD). ${ }^{*} \mathrm{P}<0.05,{ }^{* *} \mathrm{P}<0.01,{ }^{* * *} \mathrm{P}<0.001$ versus the basal value in each group. HbA1c: hemoglobin A1c; FPG: fasting plasma glucose.

group completed the 18-month study. At the end of the study, the daily dosage of sitagliptin in the SIT group had increased to $63.3 \pm 22.5 \mathrm{mg}$, while the dosage of glimepiride was $1.1 \pm$ $0.5 \mathrm{mg} /$ day. The daily dosage of glimepiride in the SU group was $2.1 \pm 1.3 \mathrm{mg}$, which was close to the initial dosage. The daily dosage of metformin was increased from $718.8 \pm 208.6$ $\mathrm{mg}$ to $816.7 \pm 371.6 \mathrm{mg}$ in the SIT group, and from $750.0 \pm$ $311.8 \mathrm{mg}$ to $781.3 \pm 340.0 \mathrm{mg}$ in the SU group. Pioglitazone was added at $15 \mathrm{mg} /$ day in one patient in the SIT group, and to two in the SU group.

As shown in Figure 2, FPG in both groups fell slightly, although not significantly, after adding sitagliptin or increasing the dose of glimepiride. Figure 2 also illustrates the changes in $\mathrm{HbA1c}$ in both groups. Although there was no difference between the two groups, there was a very significant intra-group difference $(\mathrm{P}<0.001)$. In the SIT group, HbA1c had significantly decreased from $7.8 \pm 1.1 \%$ to $7.1 \pm 0.6 \%$ at 18 months after initiation of the study $(\mathrm{P}<0.01)$, while $\mathrm{HbA} 1 \mathrm{c}$ in the SU group had fallen from $7.7 \pm 0.6 \%$ to $7.2 \pm 0.8 \%$ at the end of the study $(\mathrm{P}<0.01)$. Plasma levels of insulin, $\mathrm{CPR}$ and proinsulin did not show any changes between or within the groups during the study period (Table 2). However, as shown in Figure 3, although the ratio of PI/I did not differ significantly between the groups, the intra-group difference was significant $(\mathrm{P}<0.05)$. In the SU group only, the ratio of PI/I was lower 6 months after initiation of the study and thereafter $(\mathrm{P}<0.05)$. Regarding HOMA- $\beta$, inter-group difference was not significant, but intra-group difference reached a significant level $(\mathrm{P}<0.05)$. As illustrated in Figure 3, HOMA- $\beta$ in the SIT group had significantly increased 6 and 18 months after initiation of the study, while HOMA- $\beta$ had also increased after 6 months in the SU group. Homeostasis model assessment for insulin resistance (HOMA-R) levels did not change in either group after initiation of the study (Table 2).

In regard to urinary parameters, as shown in Table 2, intergroup difference in eGFR was not significant, but intra-group difference was $(\mathrm{P}<0.001)$. eGFR in the SIT group showed a significant decrease 12 and 18 months after start of the study, while there were no intra-group differences in the SU group. However, there were no inter-group or intra-group differences in log UACR (Table 2).

Throughout the study, body weight did not show any increases in either group (Table 2). No severe hypoglycemic episodes were seen in either group except one each in the SIT and SU group who discontinued the study as mentioned above.

\section{Discussion}

This study evaluated the long-term effects of add-on therapy of $50 \mathrm{mg}$ of sitagliptin to a very small dose of glimepiride at $1.1-1.2 \mathrm{mg} /$ day, or a small dose of glimepiride at $2.1 \mathrm{mg} /$ day, on $\beta$-cell function over 18 months. Since we could not register the planned number of patients because of difficulties 


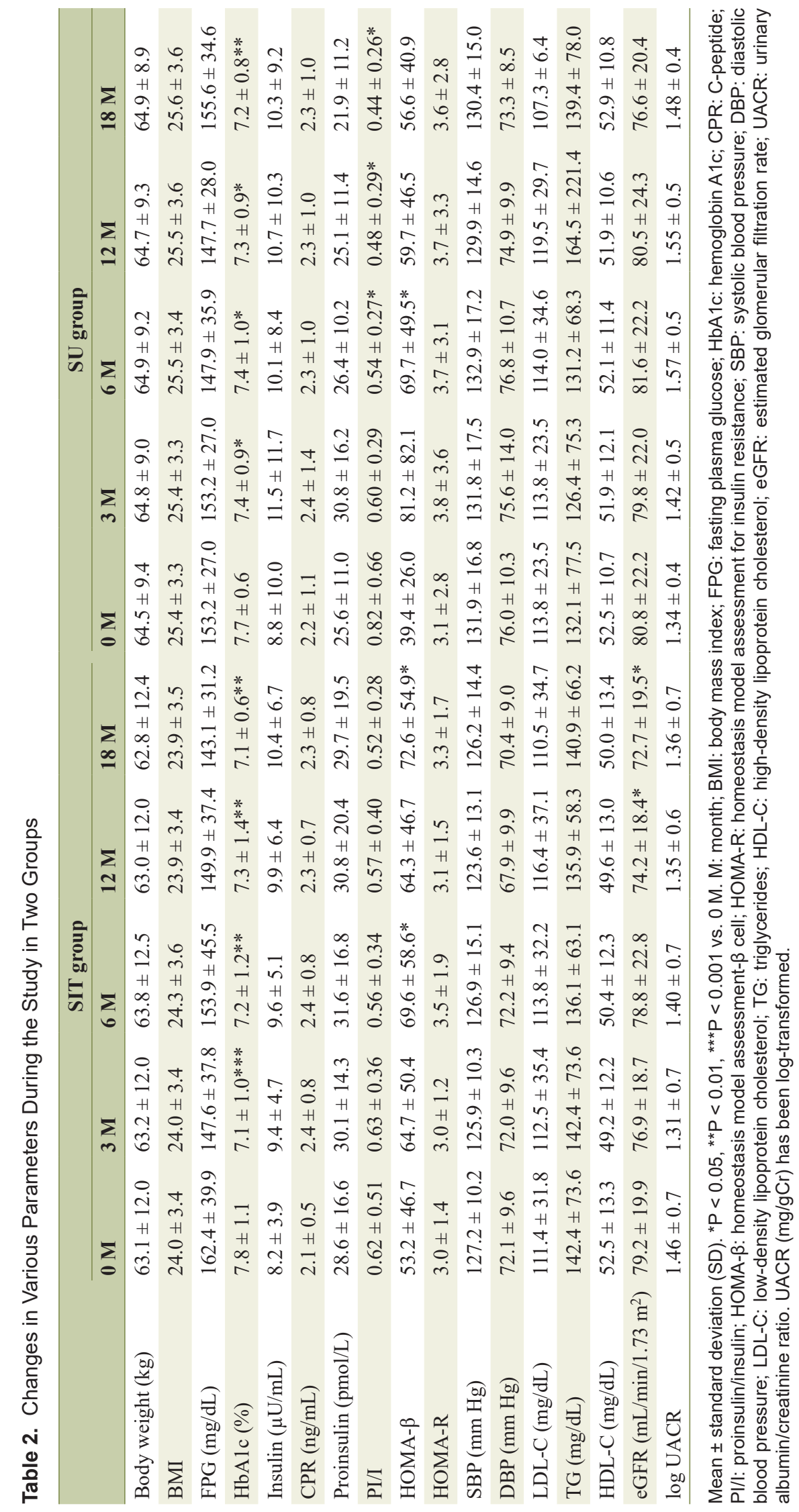



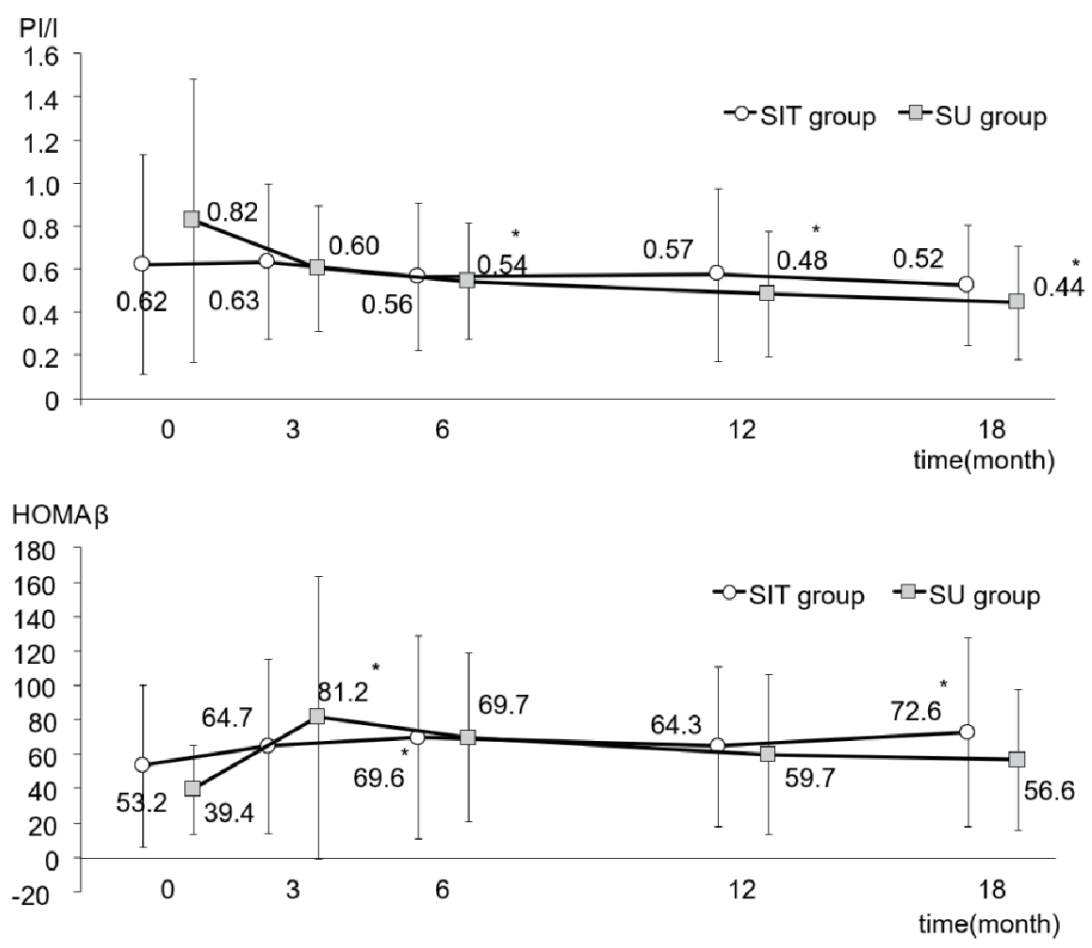

Figure 3. $\mathrm{PI} / \mathrm{I}$ ratio and HOMA- $\beta$ during the study in the SIT and SU groups. All data were presented as mean \pm standard deviation (SD). ${ }^{*} \mathrm{P}<0.05$ versus the basal value in each group. $\mathrm{PI} / \mathrm{l}$ : proinsulin/insulin; HOMA- $\beta$ : homeostasis model assessment- $\beta$ cell.

to recruit the patients treated only with SU, the power to detect the difference between two groups in effects of two regimens on $\beta$-cell function might be not sufficient. However, a significant decrease in HbAlc was observed in the group treated with add-on therapy of sitagliptin to a very small dose of glimepiride, from $7.8 \pm 1.1 \%$ at the baseline to $7.1 \pm 0.6 \%$ at the end of the study. The group treated with glimepiride at $2.1 \mathrm{mg} /$ day also showed a significant decrease in $\mathrm{HbA} 1 \mathrm{c}$, from $7.7 \pm 0.6 \%$ at the baseline, to $7.2 \pm 0.8 \%$ at the end of the study. However, $\beta$-cell secretory capacity, which was determined using plasma insulin and CPR, showed a slight increase after initiation of the study, i.e., with administration of sitagliptin or increased dose of glimepiride, but did not reach statistical significance within or between groups. HOMA- $\beta$ in the SIT group was significantly higher 6 and 18 months after initiation of the study, while HOMA- $\beta$ in the SU group was also higher 6 months after increasing the dose of glimepiride. The glucose-lowering effect of sitagliptin might therefore be due to a slight, but not significant, increase in insulin secretion from the $\beta$-cells. Factors other than insulin that might be involved in the glucoselowering effect of sitagliptin were observed in the present study, one of which was the suppressive action of glucagon with DPP-4 inhibitor, which has been demonstrated in previous studies [7-10]. Sitagliptin treatment did not increase body weight and did not cause severe hypoglycemic episodes except one mild case who discontinued the study.

On the other hand, an 18-month treatment with careful dose titration of glimepiride, the dose of which was $2.1 \mathrm{mg} /$ day, resulted in a statistically significant decrease in HbA1c without producing severe hypoglycemic episodes or weight gain. Of note was that glimepiride at a low dose did not increase plasma levels of insulin, CPR or proinsulin. This might be one of the reasons why there was no incidence of severe hypoglycemia or body weight gain except one mild case who discontinued the study. Some post-marketing reports and clinical trials with glimepiride treatment have reported a significant increase in body weight. However, these observations were noted at higher concentrations of glimepiride $(4-8 \mathrm{mg})$, titrated rapidly over a 1 - 4 week period [12]. However, in another study, the effect of another DPP-4 inhibitor, namely saxagliptin, as a 5 $\mathrm{mg}$ /day add-on to metformin, was compared with glimepiride at $1 \mathrm{mg} /$ day, titrating to a mean dose of $3.3 \mathrm{mg} /$ day add-on to metformin, in $720 \mathrm{~T} 2 \mathrm{DM}$ aged 72.6 years over 52 weeks [13]. Of the 574 participants who completed the study, the proportions of patients achieving HbAlc $<7.0 \%$ at 52 weeks without confirmed/severe hypoglycemia were similar between saxagliptin and glimepiride, or 37.9 vs. $38.2 \%$ (odds ratio (OR): 0.99, 95\% confidence interval: $0.73-1.34, \mathrm{P}=0.9415)$. Saxagliptin was superior to glimepiride in terms of primary endpoint in patients aged $<75$ years, and was numerically inferior in patients aged $\geq 75$ years. Whereas the incidence of confirmed/severe hypoglycemia was substantially higher with glimepiride than with saxagliptin, more patients treated with glimepiride achieved the HbA1c target than those treated with saxagliptin. Body weight fell by $0.8 \mathrm{~kg}$ with saxagliptin, but increased by $1.0 \mathrm{~kg}$ with glimepiride. Ferreira et al reported that sitagliptin at 50 or $25 \mathrm{mg}$ /day and glipizide at $7.7 \mathrm{mg}$ /day provided similar HbA1c-lowering effects (-0.8 vs. $-0.6 \%)$ after 
54 weeks of treatment in 426 T2DM patients with moderate to severe chronic renal insufficiency [14]. Sitagliptin was generally well tolerated, with a lower risk of hypoglycemia and weight loss versus weight gain relative to glipizide. In another study, in which the efficacy and safety of $50 \mathrm{mg}$ of sitagliptin were compared to those for T2DM patients aged $\geq 60$ years prescribed with $0.5 \mathrm{mg}$ of glimepiride for 52 weeks, and for a subsequent 52 weeks in some of the patients who were willing to continue the treatment, sitagliptin revealed non-inferiority to glimepiride in terms of changes in $\mathrm{HbA} 1 \mathrm{c}$ from the baseline at 52 weeks [15]. Sitagliptin resulted in a significantly lower rate of incidence of non-serious hypoglycemia than glimepiride. Durability of the efficacy and safety over a 2-year period of alogliptin was also compared with glipizide in type 2 diabetics with a mean age of 55.4 years [16]. HbA1c reductions at 104 weeks were $-0.68 \%,-0.72 \%$ and $-0.59 \%$ for alogliptin 12.5 and $25 \mathrm{mg}$ and glipizide $5.3 \mathrm{mg}$, respectively (both doses met the criteria for non-inferiority to glipizide $(\mathrm{P}<0.001)$, and alogliptin $25 \mathrm{mg}$ met the superiority criteria $(\mathrm{P}=0.001)$. Weight gain and incidence of hypoglycemia were greater in the glipizide group than in the groups treated with alogliptin. Taken together with the results of the present study, DPP-4 inhibitors such as sitagliptin, saxagliptin and alogliptin might be able to achieve the same degree of glycemic control as that obtained by glimepiride up to $3.3 \mathrm{mg}$ /day or glipizide up to 7.7 $\mathrm{mg}$ /day over 1 - 2 years. However, the risk of hypoglycemia and weight gain might be lower during treatment with DPP-4 inhibitors than with SUs, although the SU group using a small dose of glimepiride at $2.1 \mathrm{mg} /$ day did not show any significant increases in severe hypoglycemia or body weight. If medication cost is a major issue, the use of less expensive agent such as glimepiride, especially at a very small dose, might be an effective option as described in the Consensus Report by the American Diabetes Association (ADA) and the European Association for the Study of Diabetes (EASD) [17].

Regarding $\beta$-cell secretory capacity, Karunakaran et al [18] demonstrated a significant improvement in $\beta$-cell function with gliclazide treatment at $\leq 160 \mathrm{mg}$ /day for 1 year while demonstrating lower FPG and HbA1c without adverse effects on body weight. Gudipaty et al also reported a significant increase in $\beta$-cell secretory capacity, but only in a group treated with glimepiride titrated to about $3 \mathrm{mg} /$ day at 3 months, which was continued for another 3 months, in comparison with the group treated with exenatide at $10 \mu \mathrm{g}$ twice daily or sitagliptin at $100 \mathrm{mg}$ once a day for 6 months [19]. In the study mentioned above, Schernthaner et al observed significant increases from baseline in HOMA- $\beta$ in both treatment groups with sitagliptin and glipizide, with a greater increase seen in the glipizide group [13]. Of note in the present study is that HOMA- $\beta$ appeared to have been augmented and reached statistical significance at several time points in both groups. It seems that there was a lack of further improvement in $\beta$-cell secretory capacity with the add-on of DPP-4 inhibitor to a very small dose of glimepiride at 1.1 to $1.2 \mathrm{mg} / \mathrm{day}$, in comparison to increasing glimepiride up to $2.1 \mathrm{mg} /$ day. We can conclude that such a small dose of glimepiride may not cause a sustained insulin release from $\beta$-cells during a period of 18 months. Secondary insufficiency has been a major problem in long-term treatment with SUs at the usual dosage, such as $3-6 \mathrm{mg}$ /day of glimepir- ide, which stimulates insulin secretion from $\beta$-cells in the islets of the pancreas, resulting in an exhaustion of $\beta$-cell secretory capacities. However, our study protocol, which involved administering glimepiride at $2.1 \mathrm{mg} /$ day, might not cause secondary insufficiency over 18 months.

Of interest is the observation that the PI/I ratio showed a significant difference between the SIT and SU groups, with a significant decrease 6 months after initiation of the study and thereafter in the SU group. It has been established that the PI/I ratio reflects $\beta$-cell dysfunction associated with the onset and progression of T2DM [20, 21]. SUs at a higher dosage, or lower dosages down to $0.5 \mathrm{mg}$ of glimepiride/day, have been demonstrated to increase the PI/I ratio $[22,23]$. However, in some reports, glimepiride administered at $3 \mathrm{mg}$ /day for 6 months or at $0.5 \mathrm{mg}$ /day for 2 years did not alter the PI/I ratios $[15,19]$. It has been also reported that postprandial secretion of proinsulin was higher in T2DM patients treated with SU and metformin than in those treated with insulin glargine and metformin [24]. On the other hand, treatment with DPP-4 inhibitors with or without metformin has been reported to reduce the PI/I ratio $[15,24]$. On the contrary, in another study [19], sitagliptin at $100 \mathrm{mg} /$ day for 6 months did not change the PI/I ratio. As discussed above, effects of SUs as well as DPP-4 inhibitors on the PI/I ratio are still controversial. Although the reasons for the decreased PI/I ratio in the SU group remain to be elucidated, improved glycemic control by glimepiride might have positively affected not only the $\beta$-cell secretory capacity but also the balance between insulin production and release. Highquality comparative research is needed to investigate not only HbAlc reduction after treatment but also the durability of its effectiveness, often ascribed to longer $\beta$-cell preservation.

The present study has several limitations. First, when the study was started in 2010, the target patient number was 210 . However, the enrollment period was elongated by 1 year and the study was terminated in 2016 after enrolling 62 patients because of difficulty in patient recruitment. The paucity of participants may be explained by the fact that sitagliptin and other DPP-4 inhibitors rapidly became the first-line therapy for T2DM in Japan during that period. Second, HOMA- $\beta$ was calculated as one of indexes reflecting $\beta$-cell function. However, FPG levels of participants in the present study were more than $140 \mathrm{mg} / \mathrm{dL}$ and therefore HOMA- $\beta$ might be underestimated.

In conclusion, an add-on therapy of $50 \mathrm{mg}$ of sitagliptin to a very small dose of glimepiride at $1.1-1.2 \mathrm{mg} /$ day or a small dose of glimepiride at $2.1 \mathrm{mg} /$ day was effective in improving or maintaining the same degree of glycemic control, without deteriorating $\beta$-cell function or causing serious adverse events such as severe hypoglycemia or body weight gain over 18 months. However, further studies over a longer period may be needed to evaluate the durability of a combination therapy of DPP-4 inhibitor with a very small dose of a SU or monotherapy with a small dose of a SU such as glimepiride at about $2 \mathrm{mg} /$ day, in relation to $\beta$-cell function.

\section{Acknowledgments}

We also thank all investigators of Saitama Incretin Study 
Group. In addition to authors, physicians listed below registered the patients to Saitama Sitagliptin Study: Kiyoaki Inoue, Munemichi Inaba, Saitama Medical University Hospital, Iruma-gun, Saitama; Takafumi Tuchiya, Kenji Hara, Mariko Suetsugu, Kyoko Watanabe, Saitama Medical Center, Dokkyo Medical University, Koshigaya, Saitama; Hirosi Shuto, Minamikoshigaya Kenshinkai Clinic, Koshigaya, Saitama.

\section{Financial Disclosure}

This study was funded by the Waksman Foundation of Japan, Inc.

\section{Conflict of Interest}

Shigehiro Katayama has received honoraria from MSD Co. Ltd. The other authors declare no conflict of interest.

\section{Informed Consent}

Informed consents were obtained.

\section{Author Contributions}

YA, MM and SK conceived and designed the experiments. MH, II, SK, HO, MM, MK, TI, YA and SK enrolled the patients. $\mathrm{MH}$ and $\mathrm{SK}$ analyzed the data. $\mathrm{MH}$ and SK wrote the first draft of the manuscript. $\mathrm{MH}$ and SK contributed to the writing of the manuscript. All authors agreed with the manuscript's results and conclusions.

\section{References}

1. Turner RC, Cull CA, Frighi V, Holman RR. Glycemic control with diet, sulfonylurea, metformin, or insulin in patients with type 2 diabetes mellitus: progressive requirement for multiple therapies (UKPDS 49). UK Prospective Diabetes Study (UKPDS) Group. JAMA. 1999;281(21):2005-2012.

2. Kahn SE, Haffner SM, Heise MA, Herman WH, Holman RR, Jones NP, Kravitz BG, et al. Glycemic durability of rosiglitazone, metformin, or glyburide monotherapy. N Engl J Med. 2006;355(23):2427-2443.

3. Herman GA, Bergman A, Stevens C, Kotey P, Yi B, Zhao P, Dietrich B, et al. Effect of single oral doses of sitagliptin, a dipeptidyl peptidase-4 inhibitor, on incretin and plasma glucose levels after an oral glucose tolerance test in patients with type 2 diabetes. J Clin Endocrinol Metab. 2006;91(11):4612-4619.

4. Drucker DJ, Nauck MA. The incretin system: glucagon-like peptide-1 receptor agonists and dipeptidyl peptidase-4 inhibitors in type 2 diabetes. Lancet. 2006;368(9548):1696-1705.

5. $\mathrm{Xu} \mathrm{L}$, Man CD, Charbonnel B, Meninger G, Davies MJ,
Williams-Herman D, Cobelli C, et al. Effect of sitagliptin, a dipeptidyl peptidase-4 inhibitor, on beta-cell function in patients with type 2 diabetes: a model-based approach. Diabetes Obes Metab. 2008;10(12):1212-1220.

6. Iwamoto Y, Taniguchi T, Nonaka K, Okamoto T, Okuyama K, Arjona Ferreira JC, Amatruda J. Dose-ranging efficacy of sitagliptin, a dipeptidyl peptidase-4 inhibitor, in Japanese patients with type 2 diabetes mellitus. Endocr J. 2010;57(5):383-394.

7. Ahren B, Foley JE, Ferrannini E, Matthews DR, Zinman $\mathrm{B}$, Dejager S, Fonseca VA. Changes in prandial glucagon levels after a 2-year treatment with vildagliptin or glimepiride in patients with type 2 diabetes inadequately controlled with metformin monotherapy. Diabetes Care. 2010;33(4):730-732.

8. Muscelli E, Casolaro A, Gastaldelli A, Mari A, Seghieri G, Astiarraga B, Chen Y, et al. Mechanisms for the antihyperglycemic effect of sitagliptin in patients with type 2 diabetes. J Clin Endocrinol Metab. 2012;97(8):28182826.

9. Solis-Herrera C, Triplitt C, Garduno-Garcia Jde J, Adams J, DeFronzo RA, Cersosimo E. Mechanisms of glucose lowering of dipeptidyl peptidase-4 inhibitor sitagliptin when used alone or with metformin in type 2 diabetes: a double-tracer study. Diabetes Care. 2013;36(9):27562762.

10. Akiyama Y, Morita-Ohkubo T, Oshitani N, Ohno Y, Aso Y, Inukai T, Kakei M, et al. Decreased glucagon levels and decreased insulin secretion after sitagliptin versus mitiglinide administration with similar glycemic levels following an oral glucose load: a randomized crossover pharmaceutical mechanistic study. Diabetol Int. 2016;7(1):25-33.

11. Matsuo S, Imai E, Horio M, Yasuda Y, Tomita K, Nitta $\mathrm{K}$, Yamagata $\mathrm{K}$, et al. Revised equations for estimated GFR from serum creatinine in Japan. Am J Kidney Dis. 2009;53(6):982-992.

12. Goldberg RB, Holvey SM, Schneider J. A dose-response study of glimepiride in patients with NIDDM who have previously received sulfonylurea agents. The Glimepiride Protocol \#201 Study Group. Diabetes Care. 1996;19(8):849-856.

13. Schernthaner G, Duran-Garcia S, Hanefeld M, Langslet G, Niskanen L, Östgren CJ, Malvolti E, et al. Efficacy and tolerability of saxagliptin compared with glimepiride in elderly patients with type 2 diabetes: a randomized, controlled study (GENERATION). Diabetes Obes Metab. 2015;17(7):630-638.

14. Arjona Ferreira JC, Marre M, Barzilai N, Guo H, Golm GT, Sisk CM, Kaufman KD, et al. Efficacy and safety of sitagliptin versus glipizide in patients with type 2 diabetes and moderate-to-severe chronic renal insufficiency. Diabetes Care. 2013;36(5):1067-1073.

15. Terauchi Y, Yamada Y, Ishida H, Ohsugi M, Kitaoka M, Satoh J, Yabe D, et al. Efficacy and safety of sitagliptin as compared with glimepiride in Japanese patients with type 2 diabetes mellitus aged $>/=60$ years (START-J trial). Diabetes Obes Metab. 2017;19(8):1188-1192.

16. Del Prato S, Camisasca R, Wilson C, Fleck P. Durability of the efficacy and safety of alogliptin compared with 
glipizide in type 2 diabetes mellitus: a 2-year study. Diabetes Obes Metab. 2014;16(12):1239-1246.

17. Davies MJ, D'Alessio DA, Fradkin J, Kernan WN, Mathieu C, Mingrone G, Rossing P, et al. Management of Hyperglycemia in Type 2 Diabetes, 2018. A consensus report by the American Diabetes Association (ADA) and the European Association for the Study of Diabetes (EASD). Diabetes Care. 2018;41(12):2669-2701.

18. Karunakaran S, Hammersley MS, Morris RJ, Turner RC, Holman RR. The Fasting Hyperglycaemia Study: III. Randomized controlled trial of sulfonylurea therapy in subjects with increased but not diabetic fasting plasma glucose. Metabolism. 1997;46(12 Suppl 1):56-60.

19. Gudipaty L, Rosenfeld NK, Fuller CS, Gallop R, Schutta MH, Rickels MR. Effect of exenatide, sitagliptin, or glimepiride on beta-cell secretory capacity in early type 2 diabetes. Diabetes Care. 2014;37(9):2451-2458.

20. Roder ME, Porte D, Jr., Schwartz RS, Kahn SE. Disproportionately elevated proinsulin levels reflect the degree of impaired B cell secretory capacity in patients with noninsulin-dependent diabetes mellitus. J Clin Endocrinol
Metab. 1998;83(2):604-608.

21. Roder ME, Dinesen B, Hartling SG, Houssa P, Vestergaard $\mathrm{H}$, Sodoyez-Goffaux $\mathrm{F}$, Binder $\mathrm{C}$. Intact proinsulin and beta-cell function in lean and obese subjects with and without type 2 diabetes. Diabetes Care. 1999;22(4):609614.

22. Inoguchi T, Umeda F, Kakimoto M, Sako Y, Ishii H, Noda K, Kunisaki M, et al. Chronic sulfonylurea treatment and hyperglycemia aggravate disproportionately elevated plasma proinsulin levels in patients with type 2 diabetes. Endocr J. 2000;47(6):763-770.

23. Ohkura T, Inoue K, Fujioka Y, Nakanishi R, Shiochi H, Sumi K, Yamamoto N, et al. The proinsulin/insulin (PI/I) ratio is reduced by postprandial targeting therapy in type 2 diabetes mellitus: a small-scale clinical study. BMC Res Notes. 2013;6:453.

24. Pscherer S, Larbig M, von Stritsky B, Pfutzner A, Forst T. In type 2 diabetes patients, insulin glargine is associated with lower postprandial release of intact proinsulin compared with sulfonylurea treatment. J Diabetes Sci Technol. 2012;6(3):634-640. 